\title{
Für eine saubere Methodik
}

\author{
Forschungsleitfaden für Gesundheitsfachberufe Eine gute \\ Forschungsfrage zu bilden und die Studie daraufhin struktu- \\ riert durchzuführen, ist knifflig. Der Leitfaden „Forschung zu \\ komplexen Interventionen“ hilft dabei. Er dient Forschern \\ aus Gesundheitsfachberufen als Handbuch für eine saubere \\ Methodik. Seit März 2016 gibt es eine überarbeitete Version.
}

Welche Intervention passt besser zu einer Patientengruppe und zu welchem Ergebnis führt sie? Eine aussagekräftige Hypothese können Therapeuten mithilfe von PICOS aufstellen.

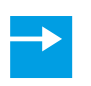

Der Leitfaden „Forschung zu komplexen Interventionen “ entstand 2013 in interdisziplinärer Zusammenarbeit von Ergound Physiotherapeuten, Pflegenden, Hebammen und Logopäden. Eine Arbeitsgruppe aus 21 Wissenschaftlern überarbeitete ihn gemeinsam mit dem Deutschen Cochrane Zentrum und unter Förderung des Bundesgesundheitsministeriums. Sie wollen damit zur Evidenzbasierung in den Gesundheitsfachberufen beitragen. Der Leitfaden dient dazu, vielversprechende Behandlungsansätze zu ermitteln und die Forschungsfrage über das sogenannte PICOS-Schema zu schärfen. Hat ein Forscher ein relevantes Gesundheitsproblem ermittelt, ist PICOS ein wichtiger Schritt, um seine wissenschaftliche Fragestellung zu untermauern.

Erster Schritt: Mit PICOS fängt die Forschung an $\rightarrow$ PICOS ist ein häufig verwendetes Hilfsschema, um eine wissenschaftliche Fragestellung auszuarbeiten und zu präzisieren. Wissenschaftler definieren darin fünf Hauptparameter, die sich international etabliert haben [1]:

$\rightarrow$ Patients: Patienten mit einem definierten Gesundheitsproblem

$\rightarrow$ Intervention: (komplexe) Maßnahme der Gesundheitsfachberufe

$\rightarrow$ Comparison: Kontrollintervention

$\rightarrow$ Outcome: Zielgröße

$\rightarrow$ Studydesign: Studiendesign
Ein Therapeut vermutet beispielsweise einen Zusammenhang, hat einen Verdacht und beschreibt das zu untersuchende Problem erst einmal in seinen Worten, zum Beispiel: „Physische Aktivität verringert Rückenschmerzen.“ Mit PICOS übersetzt er dieses eher umgangssprachlich formulierte Gesundheitsproblem in eine beantwortbare und wissenschaftliche Fragestellung:

$\rightarrow$ Wer? Patienten mit chronisch unspezifischen Rückenschmerzen

$\rightarrow$ Welche Behandlung? spezifisches Training der Bauch- und Rückenmuskulatur

$\rightarrow$ Welche Kontrollbedingung? spazieren gehen

$\rightarrow$ Welches Ergebnis? Rückgang der Schmerzen (messbar durch die VAS)

$\rightarrow$ Studiendesign? randomisierte, kontrollierte Studie (RCT)

Wichtig ist, dass der Therapeut dabei nur eine Frage stellt und diese sinnvoll und vielversprechend für einen Therapiebereich ist. Aus PICOS bildet er nun folgende Hypothese: Intervention $A$ (spezifisches

Training der Bauch- und Rückenmuskulatur) verringert im Vergleich zur Kontrollintervention B (spazieren gehen) bei der Patientengruppe XY (chronische unspezifische Rückenschmerzen) den Schmerz um beispielsweise drei Punkte auf der VAS.
Zweiter Schritt: Auf der Suche nach externer Evidenz $\rightarrow$ Im nächsten Schritt ermittelt der Forscher die Evidenzlage. Er schaut, ob es für seine Intervention bzw. Fragestellung bei der gewählten Zielgruppe bereits einen wissenschaftlichen Nachweis des Nutzens gibt (externe Evidenz). Mit den Stichwörtern aus PICOS sucht er in medizinisch wichtigen Datenbanken wie PubMed, Cochrane, Medline oder PEDro nach den besten verfügbaren Studien. Für eine effiziente Recherche sind spezifisches Fachwissen und eine gute Einarbeitung in den Suchablauf notwendig. Die Suche in den Datenbanken, die Wahl der sogenannten Keywords (Schlüsselwörter), deren Kombination und die Einstellung der Limits (Einschränkungen), dass die Schlüsselwörter zum Beispiel nur im Titel der Studien vorkommen sollen, sind enorm wichtig, um die zum Thema passende und hochwertige Literatur zu finden [2]. 

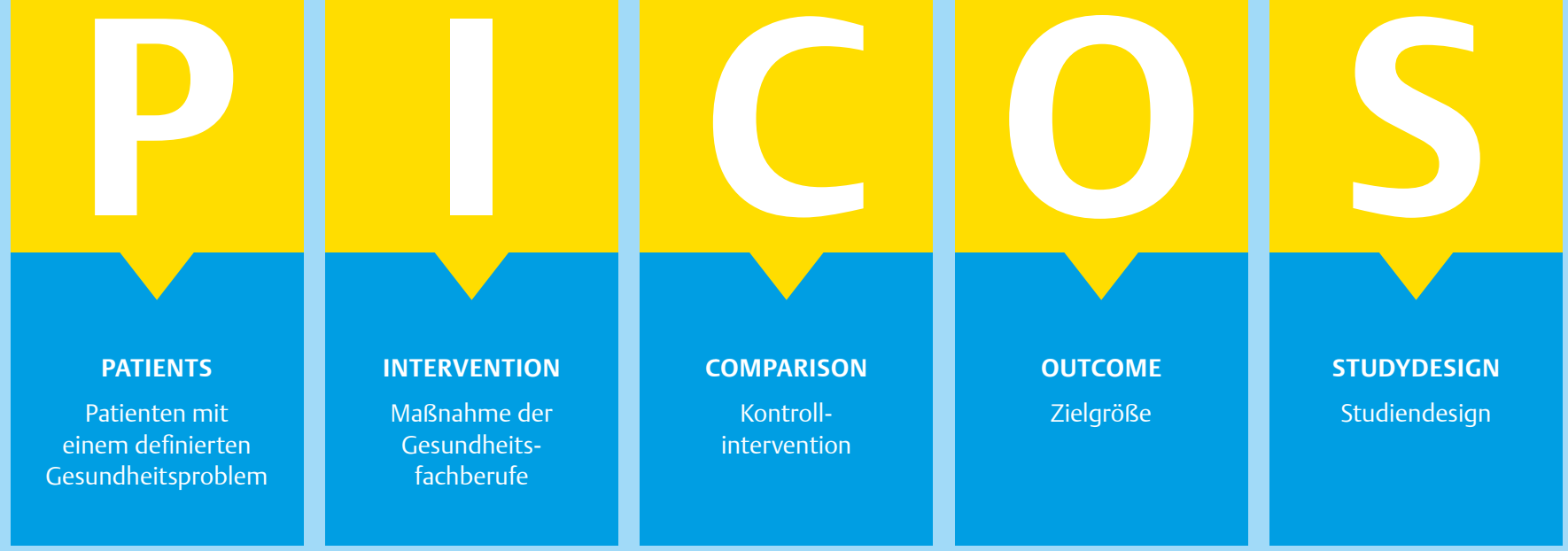

Dritter Schritt: Die Evidenzlage bewer-

ten $\rightarrow$ Im dritten Schritt beurteilt der Wissenschaftler die Evidenzlage, das heißt, er bewertet die recherchierte Literatur. Dabei ist es wichtig, auf die Qualität des Gefundenen zu achten. Die größte Aussagekraft hat ein Review (= systematische Übersichtsarbeit mehrerer Studien). In der Hierarchie absinkend folgen randomisierte, kontrollierte Studien (RCTs), Fall- und Beobachtungsberichte und an letzter Stelle die Expertenmeinung. In unserem Beispiel bedeutet das, dass der Therapeut in eine vermeintlich hochrangige Person, die behauptet, dass Spazierengehen bei Rückenschmerzen hilft, weniger Vertrauen setzen darf als in ein RCT oder Review. Diese hierarchische Abstufung nennt sich Levels of Evidence und hilft Wissenschaftlern, die Literatur und deren Schlussfolgerung einschätzen zu können. Zudem hat sich in den letzten Jahren das GRADE-System (Grading of Recommendations, Assessment, Development and Evaluation) etabliert, um die Qualität von wissenschaftlichen Arbeiten zu beurteilen (॰ ERGOPRAXIS 7-8/10, S. 14).

\section{Studienlimitationen erkennen und bei} der eigenen Arbeit vermeiden $\rightarrow$ Um eine Studie oder ein Review besser bewerten zu können bzw. selbst eine qualitativ hochwertige Studie durchzuführen, sind die Begriffe „Studienlimitationen“ bzw. „Risk of Bias“ von groBer Bedeutung. Diese sogenannte Gefahr der Verzerrung können Mängel im Studiendesign oder der -durchführung sein [3]. Wird beispielsweise gegenüber den Probanden und Therapeuten nicht geheim gehalten, ob sie in der Interventions- oder der Kontrollgruppe sind (Verblindung), gibt es Verluste bei Nachbeobachtungen. Wird nur selektiv von Endpunkten berichtet oder sind die Messinstrumente unzureichend validiert, kommt es zu Verzerrungen. Auch wie kompetent ein Therapeut ist und Fehlverhalten der Probanden können zu Limitationen führen. Das mindert das Vertrauen in die Studieneffekte.

Die Qualität von Studien sinkt zudem durch Inkonsistenz, etwa die Heterogenität der Teilnehmer, unterschiedliche Behandlungsdosierungen oder Vergleichsinterventionen bzw. widersprüchliche Ergebnisse [4]. Auch der
Publikationsbias spielt bei den Studienlimitationen eine Rolle: Er bezeichnet die Gefahr, dass wichtige Studienergebnisse nicht publiziert wurden [5]. Oft werden Studien, die positive Behandlungseffekte zeigen, schneller und vermehrt publiziert. Negative Studien hingegen weniger. Damit fehlen diese Informationen, und Behandlungseffekte werden überschätzt [5].

Forschung in der Therapie ist, wie der Name des Leitfadens schon sagt, komplex. Es gibt sehr viele Variablen, die ein Forscher bei der Erstellung oder Bewertung einer Studie beachten muss. Der Leitfaden dient dabei als „Kochbuch“. Er hilft, Hindernisse zu überwinden und die Berufsgruppen durch einen koordinierten Ausbau der Forschung voranzutreiben.

Katrin Veit

$\boldsymbol{B}$ Leitfaden „Forschung zu komplexen Interventionen" bit.ly/GFB_Leitfaden

$\boldsymbol{\rightarrow}$ Literaturverzeichnis www.thieme-connect.de/products/ ergopraxis > "Ausgabe 1/17“ 